\title{
Problem Drinking in Cancer Survivors
}

\author{
Seung-Won $\mathrm{Oh}^{*}$ \\ Department of Family Medicine, Healthcare System Gangnam Center, Seoul National University Hospital, Seoul, Korea
}

\section{See original paper on 222}

Alcohol drinking is a significant public health issue. Alcoholrelated diseases are globally responsible for 3 million annual deaths, representing $5.3 \%$ of all deaths. ${ }^{1)}$ Korea also faces several alcohol-related health problems. A typical disease associated with alcohol is cancer. Oral, pharynx, larynx, esophageal, liver, colorectal, and female breast cancer are particularly known to have causal relationships with drinking alcohol. Growing evidence shows that alcohol is associated with increased risks of melanoma and prostate and pancreatic cancers.

In the present issue, Bae et al. ${ }^{2)}$ investigated the impact of changed drinking behavior on cardiovascular risk factors among cancer survivors. In this study using the Korean National Health Insurance Service Health-Examinee Cohort Database, drinking behavior improvements, from problem drinking to moderate drinking, after a cancer diagnosis decreased body mass index, blood pressure, and cholesterol levels. Cancer survivors generally exhibit satisfactory health behaviors compared to those who have not been diagnosed with cancer. ${ }^{3)}$ Examples include smoking cessation, alcohol abstinence, and regular exercise after cancer treatment. This change in health behavior can affect the risk of cancer relapse and treatment prognosis, as well as overall health.

However, many cancer survivors maintain their unhealthy behavior, including problem drinking. In a US study on alcohol consumption among adult cancer survivors using National Health Interview Survey data, most participants with a history of cancer (56.5\%) self-reported as current drinkers, including $34.9 \%$ who exceeded moderate drinking limits and $21.0 \%$ who engaged in binge drinking. ${ }^{4)}$ In a study by Bae et al., ${ }^{2)}$ it was found that 1,197 (32\%) of the 3,692 cancer survivors who had a drinking problem before the cancer diagnosis con- tinued to drink over the cut-off of problem drinking.

For the effective health management of cancer survivors who cannot change their drinking habits, we must reflect on their reasons to continue drinking. In addition to the problem of alcohol dependence, a lack of awareness of the effects of drinking on health and socio-economic status may affect their drinking habit. According to a study using data from the Korean National Health and Nutrition Survey, it was reported that education levels and smoking are related to the risk of drinking of cancer survivors. ${ }^{5)}$ More research is needed on the factors affecting cancer survivors' health behavior. Cancer survivors' primary care practitioners should further examine patients' health-related behaviors and other factors that may affect their behavior.

Another important point is that moderate drinking of about 1-2 glasses per day can hinder the prevention of cancer. In the European Union's 2016 cancer prevention recommendations, the portion addressing drinking was revised to "If you drink alcohol of any type, limit your intake. Not drinking alcohol is better for cancer prevention." ${ }^{6)}$ In 2017, the American Society of Clinical Oncology also warned that even a small amount of alcohol could increase the risk of various cancers. ${ }^{7)}$ This reflects recent research findings indicating that drinking 1-2 glasses a day can increase the risk of cancer. The recently revised Korean cancer prevention guidelines also recommend avoiding alcohol, even in small amounts. There is still controversy over the effect of drinking a small amount of alcohol on the risk of developing cancer, therefore further research is needed, and the related research results must be examined.

\section{CONFLICT OF INTEREST}

No potential conflict of interest relevant to this article was reported. 


\section{ORCID}

Seung-Won Oh: https://orcid.org/0000-0003-3800-0754

\section{REFERENCES}

1. World Health Organization. Global status report on alcohol and health 2018. Geneva: World Health Organization; 2018.

2. Bae EM, Cho IY, Jun JH, Lee K, Kim JY, Bae WK, et al. Risk factors of cardiovascular disease according to alcohol behavioral change after cancer diagnosis. Korean J Fam Med 2020;41:222-8.

3. Oh MG, Han MA, Park J, Ryu SY, Park CY, Choi SW. Health behaviors of cancer survivors: the fourth Korea National Health and Nutrition
Examination Survey (KNHANES IV, 2007-09). Jpn J Clin Oncol 2013; 43:981-7.

4. Sanford NN, Sher DJ, Xu X, Ahn C, D'Amico AV, Aizer AA, et al. Alcohol use among patients with cancer and survivors in the United States, 2000-2017. J Natl Compr Canc Netw 2020;18:69-79.

5. Ko H, Song YM, Shin JY. Factors associated with alcohol drinking behavior of cancer survivors: the Korean National Health and Nutrition Examination Survey. Drug Alcohol Depend 2017;171:9-15.

6. Scoccianti C, Cecchini M, Anderson AS, Berrino F, Boutron-Ruault MC, Espina C, et al. European code against cancer 4th edition: alcohol drinking and cancer. Cancer Epidemiol 2016;45:181-8.

7. LoConte NK, Brewster AM, Kaur JS, Merrill JK, Alberg AJ. Alcohol and cancer: a statement of the American Society of Clinical Oncology. J Clin Oncol 2018;36:83-93. 